\title{
Use of Percutaneous Aspiration Thrombectomy vs. Anticoagulation Therapy to Treat Acute Iliofemoral Venous Thrombosis: 1-year Follow-up Results of a Randomised, Clinical Trial
}

\author{
Volkan Cakir · Aytac Gulcu • Emrah Akay • \\ Ahmet E. Capar - Tugra Gencpinar • \\ Banu Kucuk • Ozalp Karabay · A. Yigit Goktay
}

Received: 5 November 2013/Accepted: 21 April 2014/Published online: 17 June 2014

(C) Springer Science+Business Media New York and the Cardiovascular and Interventional Radiological Society of Europe (CIRSE) 2014

\begin{abstract}
Purpose The purpose of this study was to compare the efficacy of percutaneous aspiration thrombectomy (PAT) followed by standard anticoagulant therapy, with anticoagulation therapy alone, for the treatment of acute proximal lower extremity deep vein thrombosis.

Methods In this randomised, prospective study, 42 patients with acute proximal iliofemoral deep vein thrombosis documented via Doppler ultrasound examination, were separated into an interventional treatment group (16 males, 5 females, average age 51 years) and a medical treatment group (13 males, 8 females, average age 59 years). In the interventional group, PAT with largelumen 9-F diameter catheterisation was applied, after initiation of standard anticoagulant therapy. Balloon angioplasty ( $n$ 19) and stent implementation (n: 14) were used to treat patients with residual stenosis $(>50 \%)$ after PAT. Prophylactic IVC filters were placed in two patients. The thrombus clearance status of the venous system was evaluated by venography. In both the medical and interven-
\end{abstract}

V. Cakir $(\bowtie)$

Ataturk Training and Research Hospital, Department of

Radiology, Division of İnterventional Radiology, Katip Celebi

University, Izmir, Turkey

e-mail: drvolkancakir@gmail.com

A. Gulcu - A. E. Capar · B. Kucuk · A. Y. Goktay

Department of Radiology, Dokuz Eylul University Hospital,

35100 Inciraltı, Izmir, Turkey

e-mail: aytac.gulcu@deu.edu.tr
A. E. Capar
e-mail: ahmetergina@gmail.com
B. Kucuk
e-mail: banu.kucuk@deu.edu.tr

tional groups, venous patency rates and clinical symptom scores were evaluated at months 1,3 , and 12 after treatment.

Results Deep venous systems became totally cleared of thrombi in 12 patients treated with PAT. The venous patency rates in month 12 were 57.1 and $4.76 \%$ in the interventional and medical treatment groups, respectively. A statistically significant improvement was observed in clinical symptom scores of the interventional group (PAT) with or without stenting $(4.23 \pm 0.51$ before treatment; $0.81 \pm 0.92$ at month 12) compared with the medical treatment group $(4.00 \pm 0.63$ before treatment; $2.43 \pm 0.67$ at month 12). During follow-up, four patients in the medical treatment and one in the interventional group developed pulmonary embolisms.

Conclusions For treatment of acute deep vein thrombosis, PAT with or without stenting is superior to anticoagulant therapy alone in terms of both ensuring venous patency and improving clinical symptoms. PAT is a safe, inexpensive, and easily performed method of endovascular treatment 
with a low rate of major complications. Our present findings and literature data suggest that PAT can be used as first-line treatment in proximal deep vein thrombosis patients, especially when thrombolytic treatment is contraindicated.

Keywords Deep vein thrombosis - Lower extremity . Percutaneous aspiration thrombectomy . Anticoagulation treatment

\section{Introduction}

Deep vein thrombosis (DVT) is a widespread condition, and, if left untreated during early stages, may cause pulmonary embolism (PE), which has serious sequelae and is associated with a high level of mortality. The annual incidence of DVT is $5-20$ per 10,000 subjects $[1,2]$. Despite delivery of appropriate medical treatment, $1-8 \%$ of patients die from secondary PEs [3]. During long-term follow-up, postthrombotic syndrome and chronic thromboembolic pulmonary hypertension develop in 40 and $4 \%$ of patients, respectively [3].

Currently, the standard DVT therapy is prescription of low-molecular weight heparin followed by warfarin for 3-6 months. The main goal of such anticoagulation therapy is to prevent propagation of thrombi and recurrent thrombosis. However, no thrombolytic effect is evident.

Systemic thrombolysis is of little or no relevance in the context of modern IR therapy. Interventional treatments such as catheter-directed thrombolysis (CDT), percutaneous mechanical thrombectomy (PMT), and thrombus fragmentation techniques, often are used to treat DVT.

CDT and PMT aim to dissolve, mechanically fragment, and eliminate thrombi via thrombolysis. However, the rather long durations of these procedure, the risk of $\mathrm{PE}$ development during thrombectomy, venous valve damage, and cost of the devices used are major disadvantages of the procedures $[4,5]$.

Few reports of percutaneous aspiration thrombectomy (PAT) have appeared, but the technique is accepted as rapid, practicable, safe, and effective, and it affords high recanalisation rates when used to treat iliofemoral vein thrombosis [6]. Aspiration thrombectomy has some advantages compared with CDT or PMT. The former method can clear many thrombi rapidly without any risk of bleeding and eliminates the need for repeat visits to angiography units to undergo venography.

The purpose of this study was to compare the efficacy of PAT followed by standard anticoagulant therapy, with the widely accepted anticoagulation therapy (thus, medical treatment alone), for the treatment of acute lower extremity proximal DVT.

\section{Methods}

\section{Study Design}

This prospective, randomised study was performed in a University hospital between March 2010 to April 2011 after approval by the relevant Scientific Review Board and Institutional Ethics Committee. All patients were informed in detail about the purpose of the study, the medical and interventional treatment modalities to be applied, potential complications of different treatment regimes, and benefits expected from treatment. Written, informed consent was obtained from all patients. A total of 42 patients diagnosed with acute ( $<14$ days) iliofemoral-popliteal (proximal) DVT using colour Doppler ultrasound (CDUS), and who were referred to the interventional radiology department, were included. A patient was excluded if any of the following applied: presence of infrapopliteal DVT, presence of subacute or chronic DVT more than 14 days in duration, inability to lie in the prone position required for intervention, terminal systemic disease requiring palliative treatment, active bleeding (from a gastric/duodenal ulcer or the cerebrovascular system), a haemorrhagic stroke within the previous year, an impaired bleeding-clotting profile, and any haemophilic disorder, or pregnancy. Patients were randomised into two groups: aspiration thrombectomy and medical treatment.

Preprocedural CDUS examinations were performed using ultrasound equipment fitted with 7- to 12-MHz linear and 12- to 5-MHz convex probes (Philips HDI 5000 and HDI 11 ATL, Bothell, WA), and the deep venous system of the lower extremities was evaluated commencing at both common iliac veins, and continuing down to the feet. The details of any thrombus (anatomical location, distribution, level, and age) were noted. Angiographic interventions were performed using a Philips Allura Xper FD20/10 biplanar angiographic device. Guiding catheters (90-cm-long 9-F straight catheters, or catheters with $40^{\circ}$ deflections at the distal ends; Cordis Co., East Bridgewater, NJ); guidewires (hydrophilically tipped; 150- or 180-cm-long with angular ends; Terumo Co., Tokyo, Japan); 10F vascular sheaths; intravascular balloons and stents; and, (if indicated), vena cava filters, were standard materials used in endovascular procedures.

In both groups of patients, LMWH (Clexane 6000 anti$\mathrm{Xa} / 0.6 \mathrm{ml}$ bid; SC) equivalent to $120-\mathrm{mg}$ enoxaparine, and $5 \mathrm{mg}$ of warfarin sodium (Coumadin $5 \mathrm{mg} / \mathrm{d}$; PO) were given initially (thus for the first 5 days). The warfarin dose was adjusted to achieve an INR value of $2.5-3 \mathrm{IU}$, and LMWH was discontinued at the end of the fifth day. In the interventional group, aspiration thrombectomy was performed on the day of initiation of standard medical therapy (LMWH and warfarin) or on the following day. In both 
study groups, early mobilisation, walking exercises, use of $30-40 \mathrm{~mm} \mathrm{Hg}$ compression stockings, and leg elevation were recommended to all patients.

\section{Percutaneous Aspiration Thrombectomy}

Before PAT, a vena cava filter was placed in the infrarenal vena cava segment if a free mobile thrombus (detected using CDUS or venography) was present at the level of the common iliac veins or the inferior vena cava (IVC), and if the presence of a pulmonary embolism was confirmed. The contralateral common femoral vein or the right jugular vein was used as the access route for placement of the IVC filter.

PAT procedures were performed under local anaesthesia delivered to the popliteal fossa (subcutaneous $2 \%[\mathrm{w} / \mathrm{v}]$ lidocaine injection). Popliteal vein access was attained using a micropuncture set (Cook, Bloomington, IN) and a 10-F vascular sheath was placed over a 0.035 -inch-diameter guidewire under fluoroscopic guidance. Ascending venographic images of the deep venous system were obtained by administration of a non-ionic contrast agent through the sheath, and all patients received 5,000 IU heparin IV at this stage. During the procedure, a maintenance dose of 1,000 IU heparin/h was given via the IV route.

In the first step, a 90-cm-long, 9-F, straight-ended guiding catheter (Vista Brite, Cordis) was advanced through the sheath and moved to-and-fro within the 1-5$\mathrm{cm}$-long thrombotic segment, in the cephalic direction, while negative pressure was applied via the catheter hub (using a 20-ml syringe) to aspirate thrombi. Aspiration was terminated when free blood flowed into the syringe. After thrombus-free lumen status was established by venography, balloon venoplasty was performed on stenotic ( $>50 \%$ narrowed) segments. Metallic stents and post-stenting balloon dilatation were used only if significant residual stenosis was evident after balloon venoplasty or if MayThurner syndrome was present. At the end of the interventional procedure, the entry site was compressed to stop bleeding and an appropriate dressing was applied. Patients were observed for at least $2 \mathrm{~h}$ after the procedure and discharged after ensuring stability of vital signs. All patients in the PAT group were evaluated by CDUS examination performed on the third postoperative day. If recurrent thrombosis or in-stent thrombosis was evident, patients underwent secondary endovascular interventions.

Technical success was defined as patency of a clot-free lumen and restoration of free venous blood flow as demonstrated venographically.

\section{Outcome Parameters}

Demographic data on patients, risk factors for DVT, and the distributions of thrombotic segments were recorded.
Table 1 Clinical symptom scores

\begin{tabular}{lll}
\hline Symptom & Present & Absent \\
\hline Swelling on the extremity & 1 & 0 \\
Painful extremity & 1 & 0 \\
Pitting edema & 1 & 0 \\
Skin rash & 1 & 0 \\
Paresthesia & 1 & 0 \\
Restricted movement & 1 & 0 \\
\hline
\end{tabular}

The technical success rate in the interventional group was noted.

Clinical scoring was used to compare clinical symptoms and patient findings between the groups. We sought to evaluate objectively clinical symptoms using six criteria based on symptoms and signs, and attainment of each criterion scored one point. The criteria were used both at the time of diagnosis and during follow-up. Higher scores reflect "clinical worsening," whereas lower scores indicate "clinical improvement" or an "asymptomatic state" (Table 1). At the time of diagnosis, and at the 1-, 3-, and 12-month followups, the intra- and intergroup responses to treatment were compared using the clinical symptom scaling system.

\section{Statistical Analysis}

Data were analysed using the Statistical Package for Social Sciences (SPSS) software (version 16.0 for Windows). Any difference associated with a probability of 0.05 or less was considered to be statistically significant. Demographic data were evaluated using the Chi squared Pearson test and the significance of intergroup differences in variables was determined using the Mann-Whitney $U$ test. Friedman's test was used to compare the distributions of more than two variables. If significant results were obtained, the distribution of the two related variables was assessed using Wilcoxon's test.

\section{Results}

We enrolled a total of 42 acute DVT patients with iliofemoral-popliteal (proximal) thrombi and randomised them into two groups: a conventional medical treatment group and a PAT group (each with 21 patients). All patients were followed-up for 12 months both clinically and radiologically.

Mean patient age in the interventional group was 53 years ( 15 males and 6 females ranging in age from 34 to 79 years), and the mean patient age in the medical treatment group was 59 years (13 males and 8 females, age range $30-83$ years). There were 6 and 15 right- and leftside thrombosed lower extremities, respectively, in the 
interventional group and 11 and 10 in the medical treatment group. In both groups, total thrombus occlusion were detected commencing at the popliteal vein and continuing up to the iliac vein $(n=13$ and $n=8$ in the interventional and medical treatment groups, respectively), or at the femoropopliteal vein $(n=8$ and $n=13)$. In the interventional group, two patients had thrombi extending up to the IVC. No statistically significant difference between the two groups was evident in terms of age, the distribution of extremities with DVT, or the levels of thrombosed segments (all $p$ values $>0.05$ ).

In the interventional group, use of PAT alone (thus without the adjunctive procedures of PTA or stenting) allowed complete venous patency to be attained by only two patients. PTA was performed in 19 patients because $>50 \%$ residual stenosis was evident. After PTA, stents were implanted in 14 patients because of residual stenosis (iliac vein, $n=13$; femoral vein, $n=1$; Table 2). The technical success rate was $90.4 \%$. In two patients with residual stenosis, recurrent thrombosis developed on the third postinterventional day. Second interventions were performed. CDUS performed 3 days later revealed thrombosis in the patient with the femoral vein stent, whereas partial patency was achieved by the patient with the iliac vein stent. Temporary IVC filters were implanted in two patients with mobile thrombi at the level of the common iliac vein or the IVC (1 patient in either category). In the PAT group, one patient $(4.8 \%)$ developed recurrent DVT and PE $(4.8 \%)$ in the first month after treatment, caused by inappropriate termination of anticoagulant therapy. In the medical treatment group, four patients developed PEs within the first month after treatment commenced although their INR values were within therapeutic range.

During the follow-up period, significantly higher patency rates were evident in the interventional group at 1,3 , and 12 months (all $p$ values $<0.001$ ) compared with the medical treatment group (Table 3). In terms of in-stent patency rates, recurrent thrombi were detected at 1 month in a single patient with a femoral vein stent and at 3 months in another patient with an iliac vein stent (Table 4). No other incident of in-stent thrombosis was detected during the 12 months of follow-up.

At the time of initial diagnosis, the mean clinical symptom scores of the interventional and medical treatment groups were $4.23 \pm 0.51$ and $4.00 \pm 0.63$, respectively, and no significant intergroup difference was apparent in terms of symptom severity $(p>0.05)$. In comparison, significant differences (highlighting the efficacy of PAT) were evident at 1,3 , and 12 months of follow-up (all $p$ values $<0.001$ ). Compared with the medical group, the PAT group also showed a statistically significant

Table 2 Patency rates

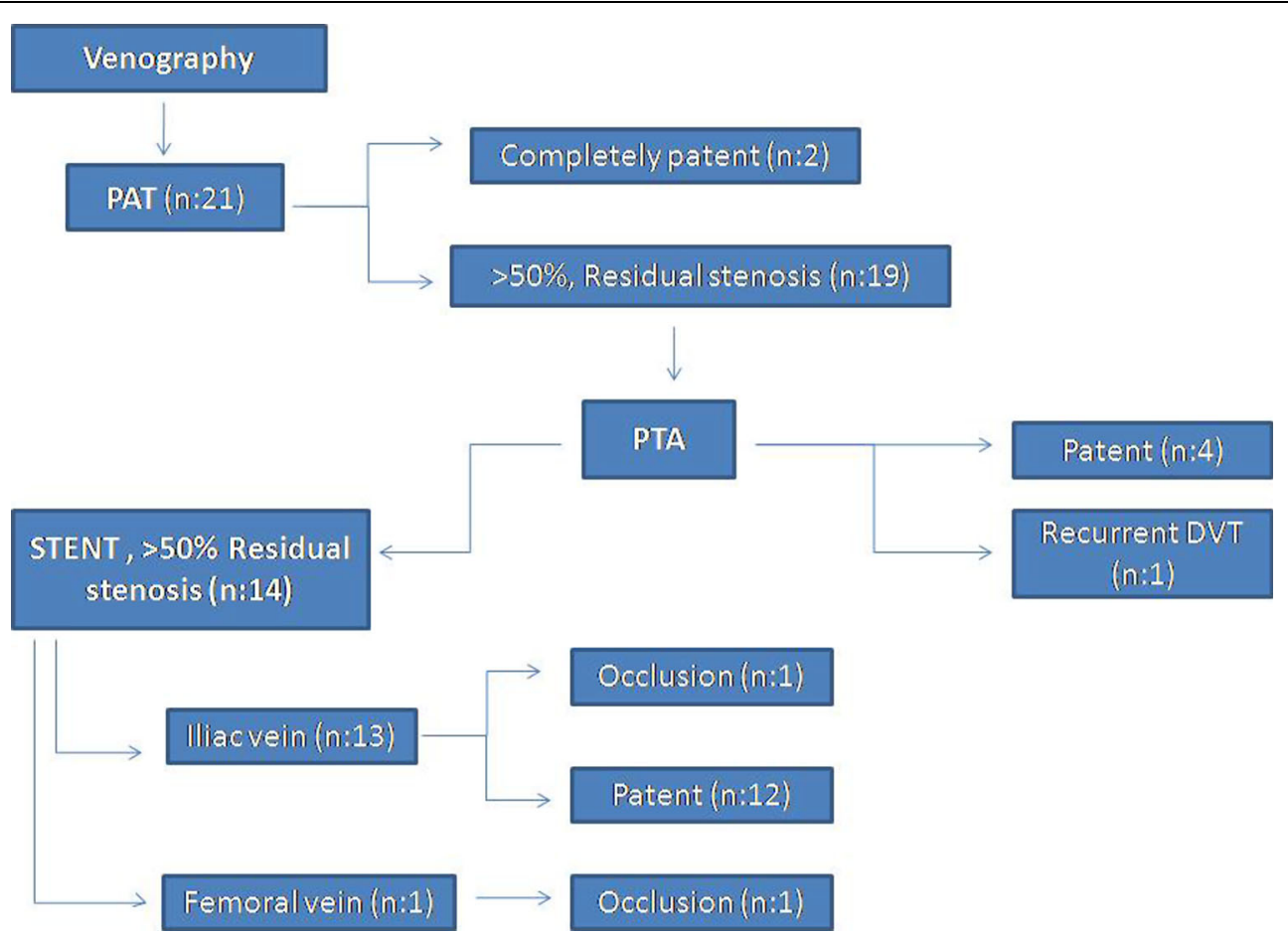

* Two patients developed stent thrombosis, one patient recurrent DVT as described in "Results" section 
Table 3 Follow-up CDUS results

\begin{tabular}{|c|c|c|c|c|c|c|}
\hline & \multicolumn{2}{|l|}{ First month } & \multicolumn{2}{|l|}{ Third month } & \multicolumn{2}{|l|}{ 12th month } \\
\hline & $\operatorname{PAT} n(\%)$ & Medical $n(\%)$ & $\operatorname{PAT} n(\%)$ & Medical $n(\%)$ & $\operatorname{PAT} n(\%)$ & Medical $n(\%)$ \\
\hline Completely patent & $13(61.9 \%)$ & 0 & $12(57.1 \%)$ & 0 & $12(57.1 \%)$ & $1(4.76 \%)$ \\
\hline Partial patency & $7(38.1 \%)$ & $5(23.8 \%)$ & $8(38.1 \%)$ & $6(28.6 \%)$ & $8(38.1 \%)$ & $15(71.4 \%)$ \\
\hline \multirow[t]{2}{*}{ Thrombosis } & $1(4.8 \%)$ & $16(76.2 \%)$ & $1(4.8 \%)$ & $15(71.4 \%)$ & $1(4.8 \%)$ & $5(23.8 \%)$ \\
\hline & $p<0.001$ & & $p<0.001$ & & $p<0.001$ & \\
\hline
\end{tabular}

Table 4 Stent patency during the follow-up

\begin{tabular}{llll}
\hline & First month & Third month & 12th month \\
\hline Complete patent & $10(71.4 \%)$ & $10(71.4 \%)$ & $10(71.4 \%)$ \\
Partial patent & $3(21.4 \%)$ & $2(14.3 \%)$ & $2(14.3 \%)$ \\
Thrombosis & $1(7.2 \%)$ & $2(14.3 \%)$ & $2(14.3 \%)$ \\
Total & $14(100 \%)$ & $14(100 \%)$ & $14(100 \%)$ \\
\hline
\end{tabular}

improvement in clinical symptom scores during the course of 12-month follow-up (Table 5).

We present venographic and CDUS images taken of one of our patients, showing pre-procedural thrombosed veins and the post-procedural follow-up results (Fig. 1).

\section{Discussion}

The main purpose of any DVT treatment is to improve symptoms and to prevent the development of PE and postthrombotic syndrome, by eliminating thrombi. Even after appropriate medical therapy, DVT may cause serious complications such as PE (10\% of patients) and postthrombotic syndrome (40\%) [2]. Despite these risks of major complications and potentially permanent sequelae, no effective treatment modality for DVT yet exists. The main aims of anticoagulant therapy are to prevent progression of existing thrombi and to lower the incidence of PE by preventing development of recurrent thrombosis. Anticoagulants do not exert a recanalisation activity. Recanalisation occurs by natural thrombus resorption over time. Many studies have reported that anticoagulation therapy prevented progression of popliteal and tibial vein thrombosis and allowed development of near-complete recanalisation in $95 \%$ of patients. However, recanalisation rates were poor $(20 \%)$ in patients with iliofemoral vein thrombosis [7].

Systemic thrombolytic therapy is markedly superior to anticoagulation therapy (using heparin) in terms of reestablishment of venous blood flow [8]. Thrombolytic agents resolve only thrombi with which they come into contact. Thus, if venous occlusion is complete, such agents sometimes do not penetrate blood clots, and treatment failure may result. The most significant concern of thrombolytic therapy is the increased risk of bleeding. Although the efficacy of thrombolytic therapy used to treat DVT is widely acknowledged; bleeding, the potential development of serious related complications, uncertainties in terms of dosage and route of administration, the requirement for admission to the intensive care unit, a prolonged hospitalisation period, and the need to conduct numerous laboratory tests to monitor health status, all indicate that this therapeutic modality is associated with limited indications.

As another therapeutic alternative, surgical thrombectomy, can be used to treat a limited number of patients and is especially preferred in patients with phlegmasia caerulea dolens [9]. However, even in patients with this rare pathological abnormality, it is not possible to achieve adequate venous patency, with preservation of venous valvular function, using this technique. Acceptable recanalisation rates have been reported using other endovascular therapeutic methods, such as CDT and PMT [7, 10, 11]. Although CDT is effective, the technique is not used widely because infusion times are long (associated with longer hospital stays) and systemic bleeding complications can occur. PMT has not received general acceptance

Table 5 Clinical symptom score results at the time of diagnosis and during the follow up for both groups

\begin{tabular}{|c|c|c|c|c|c|c|c|c|}
\hline \multirow{2}{*}{$\begin{array}{l}\text { Clinical symptom } \\
\text { score }\end{array}$} & \multicolumn{2}{|l|}{ At diagnosis } & \multicolumn{2}{|l|}{ First month } & \multicolumn{2}{|l|}{ Third month } & \multicolumn{2}{|l|}{ 12th month } \\
\hline & PAT $n(\%)$ & $\begin{array}{l}\text { Medical } \\
n(\%)\end{array}$ & PAT $n(\%)$ & $\begin{array}{l}\text { Medical } \\
n(\%)\end{array}$ & PAT $n(\%)$ & $\begin{array}{l}\text { Medical } \\
n(\%)\end{array}$ & $\operatorname{PAT} n(\%)$ & $\begin{array}{l}\text { Medical } \\
n(\%)\end{array}$ \\
\hline $\begin{array}{l}\text { Mean } \\
p \text { value }\end{array}$ & $\begin{array}{l}\mathbf{4 . 2 3} \pm 0.51 \\
p>0.05\end{array}$ & $\mathbf{4 . 0 0} \pm 0.63$ & $\begin{array}{l}\mathbf{1 . 7 7} \pm 0.59 \\
p<0.001\end{array}$ & $\mathbf{3 . 7 1} \pm 0.71$ & $\begin{array}{l}\mathbf{1 . 0 8} \pm 0.83 \\
p<0.001\end{array}$ & $\mathbf{2 . 8 6} \pm 0.79$ & $\begin{array}{l}\mathbf{0 . 8 1} \pm 0.92 \\
p<0.001\end{array}$ & $\mathbf{2 . 4 3} \pm 0.67$ \\
\hline
\end{tabular}




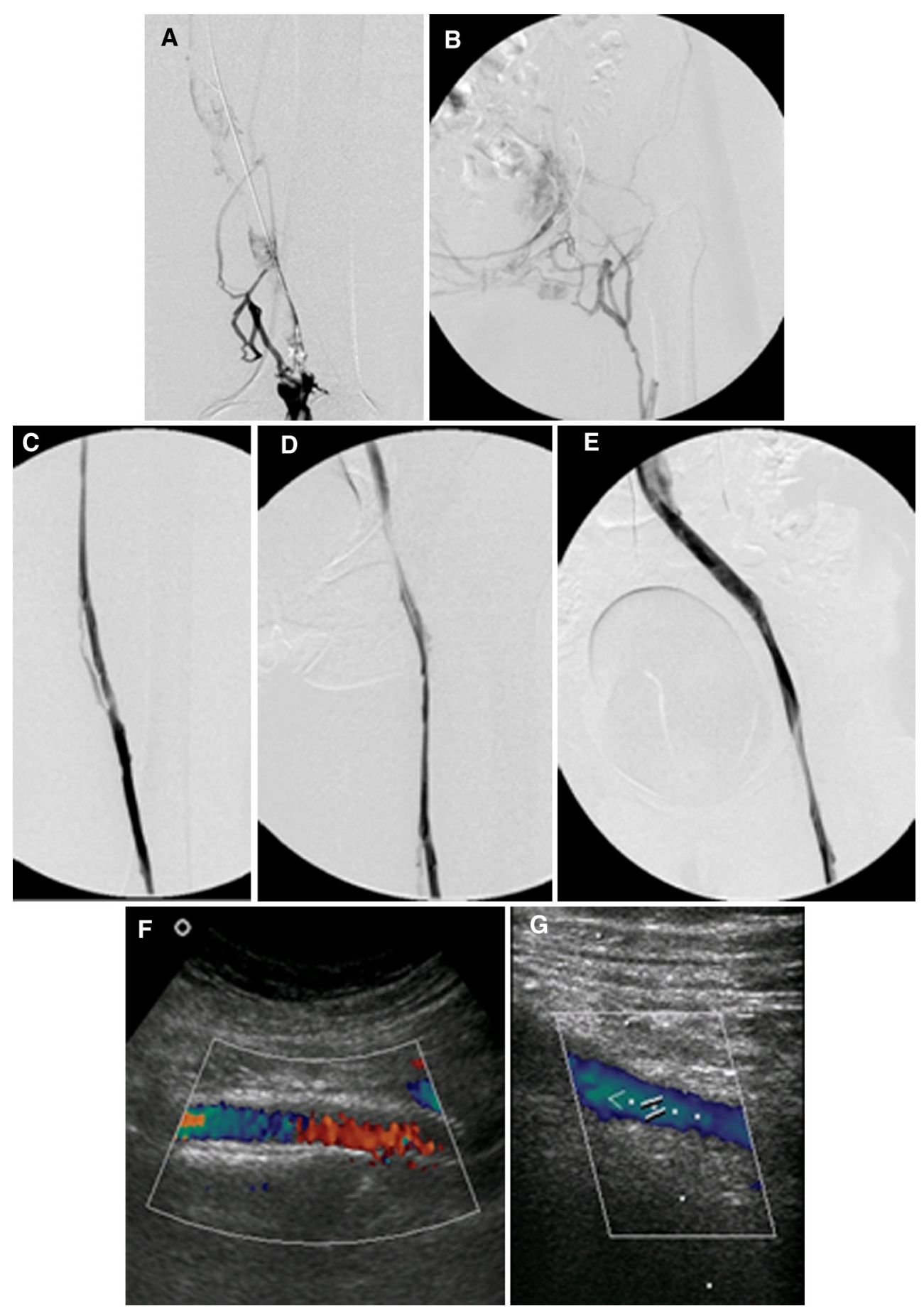

Fig. 1 Physical examination of a 40-year-old female patient revealed pain on palpation, swelling, redness, an increased diameter and pitting oedema of the left extremity, and restricted physical activity. The Homans test was positive. Her clinical symptom score was 4. Venography revealed filling defects secondary to intraluminal DVT from the level of the left popliteal vein up to the common iliac vein,

because of the associated risk of PE and damage to venous valves caused by thrombectomy devices. Also, both CDT and PMT devices are very expensive compared with PAT, angioplasty, and stenting. and involving the collateral veins $(\mathbf{A}, \mathbf{B})$. Venous patency was achieved after PAT and stenting of the left common iliac vein stenosis (C, D, E). At the 12-month follow-up visit, vascular patency was evident from the level of the popliteal vein up to the common iliac vein, with patent iliac vein stenting $(\mathbf{F}, \mathbf{G})$, and the clinical symptom score was 0

Percutaneous aspiration thrombectomy has been routinely used to effectively eliminate thrombi located in haemodialysis fistulae. Turmel-Rodrigues et al. [12] showed that the patency rate was $86 \% 6$ months after 
PAT. PAT also has been accepted as a rapid, safe, and effective method of management of iliofemoral vein thrombosis and affords higher recanalisation rates than alternative treatments [6]. However, few clinical studies have been performed. Kwon et al. [13] reported a recanalisation rate of $88.9 \%$ in 27 patients who had undergone PAT; no complication was noted. Mechanical thrombectomy devices are not required during PAT and, therefore, the risk of trauma to the vascular wall and valves is thought to be low. Thrombolytic agents are not given to PAT patients, and the bleeding complications associated with systemic therapy are therefore absent [13]. No standardised PAT aspiration method has yet been developed. However, Oguzkurt et al. [14] have described a thrombectomy method ("to and fro" manipulation while aspiration) affording rapid and effective aspiration of thrombi. We used this technique in our interventions. Our technical success rate is similar to that of previous studies using catheter aspiration in the course of PAT.

In our present study, we also evaluated clinical symptoms. In contrast, earlier studies did not use clinical scoring systems; assessments were based on subjective criteria. The mean clinical symptom score of patients in the interventional group was lower than that of the medical group $(p<0.001)$ (Table 5). Thus, PAT not only affords higher recanalisation rates but also dramatically improves clinical symptoms in patients with iliofemoral DVT. Our results show that PAT is more effective than standard anticoagulant therapy.

Many studies on implantation of IVC filters in DVT patients, to prevent PEs, have appeared. Development of minor thrombotic embolisms during endovascular treatment, without ensuing symptomatic PEs, has been reported previously. Also, implantation of IVC filters has been recommended for patients with proximal DVT for whom aggressive thrombectomy is planned, or who have mobile thrombi in proximal veins [15]. Our aspiration thrombectomy commences at the distal segments and proceeds proximally until we attain the most proximal regions of thrombi. Thus, elimination of residual distal thrombi using careful aspiration at iliac levels allows us to avoid triggering massive PEs. However, we use IVC filters if thrombi extend into the ostium of the common iliac vein, or if a mobile thrombus extends into the IVC. We experienced no PE complication using our clinical approach.

Although the number of patients who developed PE in the medical treatment group was somewhat higher than in the interventional group, the difference was not statistically significant $(p>0.05)$. However, the results suggest that PAT is associated with a lower risk of PE than is standard anticoagulant therapy.

The incidence of venous stenosis was previously reported to be $85 \%$ in patients with acute proximal DVT
[13]. In our present study, vascular stenosis was detected in $90.4 \%(n=21)$ of patients who had undergone PAT. Similar to what was found in a previous study [13], the patency rates in patients with stents were $92.8 \%$ at 1 month follow-up, and $85.7 \%$ at 3 and 12 months (Table 4). In our present study, thrombosis developed during the early postoperative period in only one iliac and one femoral vein that were stented. Stenting of the stenotic venous segments that are crossing the hip or knee joint does not ensure durability of patency. Currently, femoral vein stenting is thought to be associated with a risk of thrombosis. Therefore, repetitive balloon dilatation may be more effective when used to treat these types of stenotic lesions, and we no longer use stents to treat femoral vein stenosis.

Because our follow-up period was limited to 12 months, we could not evaluate development of postthrombotic syndrome and chronic venous insufficiency. Therefore, we cannot comment on the incidence of these conditions in patients treated with PAT. However, we continue to collect prospective data and plan to conduct a long-term followup.

\section{Conclusions}

PAT (with stenting if needed) is safe and effective method when used to treat proximal DVT. The greatest advantage of PAT is the potential to eliminate adequate numbers of thrombi without any bleeding complications. PAT can be performed as an outpatient procedure with low major complication rates. Our results are comparable to those of previous interventional studies and our technique is superior to medical treatment of proximal DVT. In conclusion, findings in our study suggest that PAT can be used as a alternative treatment in proximal DVT patients.

Conflict of interest All authors declare that no financial support, either directly or indirectly, received from any third party, to complete this work. None of the authors has any financial relationships with entities in the bio-medical arena that could be perceived to influence the usage of the modalities that are being described in the submitted work.

\section{References}

1. Fowkes FJ, Price JF, Fowkes FG (2003) Incidence of diagnosed deep vein thrombosis in the general population: systematic review. Eur J Vasc Endovasc Surg 25(1):1-5

2. Geerts W et al (2005) Prevention of venous thromboembolism. Chest 128(5):3775-3776

3. Scarvelis D, Wells PS (2006) Diagnosis and treatment of deepvein thrombosis. CMAJ 175(9):1087-1092

4. Vedantham S et al (2002) Lower extremity venous thrombolysis with adjunctive mechanical thrombectomy. J Vasc Interv Radiol 13(10):1001-1008 
5. Sharafuddin MJ et al (1999) Injury potential to venous valves from the Amplatz thrombectomy device. J Vasc Interv Radiol 10(1):64-69

6. Comerota AJ, Paolini D (2007) Treatment of acute iliofemoral deep venous thrombosis: a strategy of thrombus removal. Eur $\mathbf{J}$ Vasc Endovasc Surg 33(3):351-360 discussion 361-362

7. Elsharawy M, Elzayat E (2002) Early results of thrombolysis vs anticoagulation in iliofemoral venous thrombosis. A randomised clinical trial. Eur J Vasc Endovasc Surg 24(3):209-214

8. Rogers LQ, Lutcher CL (1990) Streptokinase therapy for deep vein thrombosis: a comprehensive review of the English literature. Am J Med 88(4):389-395

9. Plate $\mathrm{G}$ et al (1984) Thrombectomy with temporary arteriovenous fistula: the treatment of choice in acute iliofemoral venous thrombosis. J Vasc Surg 1(6):867-876

10. Shi HJ et al (2009) Percutaneous mechanical thrombectomy combined with catheter-directed thrombolysis in the treatment of symptomatic lower extremity deep venous thrombosis. Eur $\mathbf{J}$ Radiol 71(2):350-355
11. Bush RL et al (2004) Pharmacomechanical thrombectomy for treatment of symptomatic lower extremity deep venous thrombosis: safety and feasibility study. J Vasc Surg 40(5):965-970

12. Turmel-Rodrigues L et al (2001) Manual catheter-directed aspiration and other thrombectomy techniques for declotting native fistulas for hemodialysis. J Vasc Interv Radiol 12(12):1365-1371

13. Kwon SH et al (2009) Percutaneous aspiration thrombectomy for the treatment of acute lower extremity deep vein thrombosis: is thrombolysis needed? Clin Radiol 64(5):484-490

14. Oğuzkurt L, Ozkan U, Gülcan O, Koca N, Gür S (2012) Endovascular treatment of acute and subacute iliofemoral deep venous thrombosis by using manual aspiration thrombectomy: long-term results of 139 patients in a single center. Diagn Interv Radiol 18(4):410-416

15. Noguchi $M$ et al (2003) Thrombus removal with a temporary vena caval filter in patients with acute proximal deep vein thrombosis. Heart Vessels 18(4):197-201 